\title{
Analysis and manufacturing of bistable metallic profiles
}

Received: 19 October 2020 / Accepted: 10 February 2021 / Published online: 6 March 2021 (C) The Author(s) 2021

\begin{abstract}
Bistable metal sheets with a coiled transport geometry and an unfolded profile as second stable state, are of great interest as lightweight components. It is well known that a specific distribution of residual stresses is necessary to enable bistable properties. With the help of numerical FE models, the optimal process parameters for production of such sheets are evaluated. Afterwards, incremental bending and roll forming experiments are conducted in order to analyse possible continuous production methods for bistable metallic profiles. Finally, the influence of bending and rolling radii on bistability and profile geometries in stable states are investigated.
\end{abstract}

Keywords Residual stress $\cdot$ Finite element method (FEM) $\cdot$ Bistable profile $\cdot$ Roll forming

\section{Introduction}

Residual stresses occur in components manufactured by forming technology [1]. They are usually undesirable and must be controlled to a certain extent [2]. Nevertheless, there are components, which only show their functional properties due to an exact distribution of the residual stresses. In general, a bent sheet of metal reaches a mechanical equilibrium after spring back if the resulting bending moments caused by introduced residual stresses are tends to zero [3]. However, residual stresses can be introduced into the sheet by a certain sequence of different forming processes so that two stable states exist. A well-known example is the tape measure, which has two stable configurations: (1) the coiled transport geometry and (2) the unfolded measuring geometry [4], which can both be seen in Fig. 1a. The tape measure is produced by bending and subsequent roll forming (only one single pass) and exhibits only a slightly curved section in the measuring configuration. Accordingly, it's structural stiffness strongly depends on the loading direction. To overcome this limitation, the authors showed in earlier work, that the combination of two incremental bending processes along two orthogonal axes in two opposite directions (see Fig. 2a) allow for the production of a fully closed and bistable profile, which can be seen in Fig. 1b [6]. However, as the tool length limits the width of the bending operation, this process does not allow for longer bistable profiles. This limitation can be overcome by using a roll forming process in the longitudinal direction. In [7] it is shown that monostable tubular profiles can be produced using thin steel sheets with a high yield strength.

Bistable metallic profiles would offer a compact transport geometry, could be unfolded on installation site and would not require any housing at all. This results in various sophisticated application possibilities such as the unfolding of solar panels in outer space satellites (weight advantage through omission of the housing) [8] or the fast assembly of structures [9].

P. Pavliuchenko· G. Hirt· M. Teller

Institute of Metal Forming (IBF), RWTH Aachen University, Intzestr. 10, 52072 Aachen, Germany

E-mail: pavlo.pavliuchenko@ibf.rwth-aachen.de 


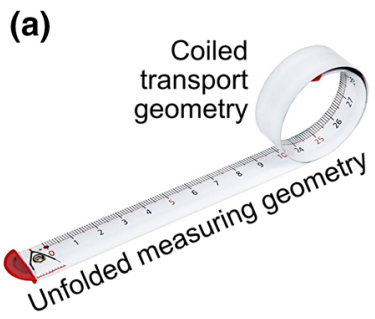

(b)

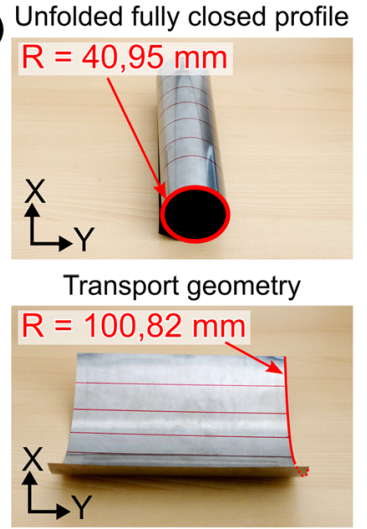

Fig. 1 Examples for bistable profiles: a tape measure with slight curvature [5] and b fully closed bistable profiles [6]

For the prediction of bistable properties and profile radii on laboratory scale (small sheets), numerical [6] and semi-analytical models [10] have been developed. However, these models assume only single bending of small [6] or infinite samples [10] along the bending radius. Furthermore, in order to achieve large area bistable profiles, several bending operations are required, which cannot be represented by the models mentioned.

Hence, the goal of this paper is analysis and manufacturing of large area bistable metallic profiles by simulation and experiment in order to gain a first insight to the possibility of producing bistable sheets by different process routes. With newly developed numerical models, the general influence of different production parameters (i.e. bending radii) and material properties (i.e. yield strength) on bistability and final profile geometry are investigated. Due to the high experimental costs, the simulations cannot be fully validated, but basic manufacturing potentials of both considered routes can be approximated by simulation.

\section{Considered process strategies}

As already explained above, there are two basic manufacturing strategies known, which can produce small (lab-scale) bistable profiles with different radii of curvature: bending twice incrementally along orthogonal axes or incremental bending followed by roll forming in one pass. As the bending radius in the roll forming pass must be much smaller than desired radius of the unfolded geometry, this process is limited to circular sections like in the tape meter shown. Based on this state of the art, two process routes for the production of large area bistable profiles are considered promising: (1) the already mentioned sequence of incremental die bending operations along two orthogonal axes in two opposite directions with specific bending radii (PR1, Fig. 2a). Although the maximum profile size is limited by the tool length, only one production machine is required for this process route. (2) Production route PR2 (Fig. 2b) consists of an initial incremental bending along the shorter side of the sheet and subsequent roll forming in orthogonal direction within several passes. Due to this procedure the bending tool length only limits the profile width, and the profile length is theoretically infinite. However, this process route requires two production machines.

Besides the production routes, the influence of the material (especially the flow curve) must also be considered. With the same number of bending operations (incremental bending as well as roll forming passes), the springback and thus the final profile geometry is different depending on the material strength. Therefore, two steels with two different initial yield strength (1.1274 with $1690 \mathrm{MPa}$ and 1.4310 with $870 \mathrm{MPa}$, see Fig. 3) and comparable strain hardening are used to investigate this influence.

\section{Simulation study on the different production routes}

An attempt to simulate such processes may face certain challenges. Those are e.g. the need to simulate deformation of a thin sheet with a high yield strength, demand for stabilization of sheet during incremental bending and rolling, the problem of transfering residual stress and strain values from one simulation step to another etc. Nevertheless, a sufficiently accurate FE-model would help to identify a suitable production window for the real manufacturing of bistable metallic sheets. 


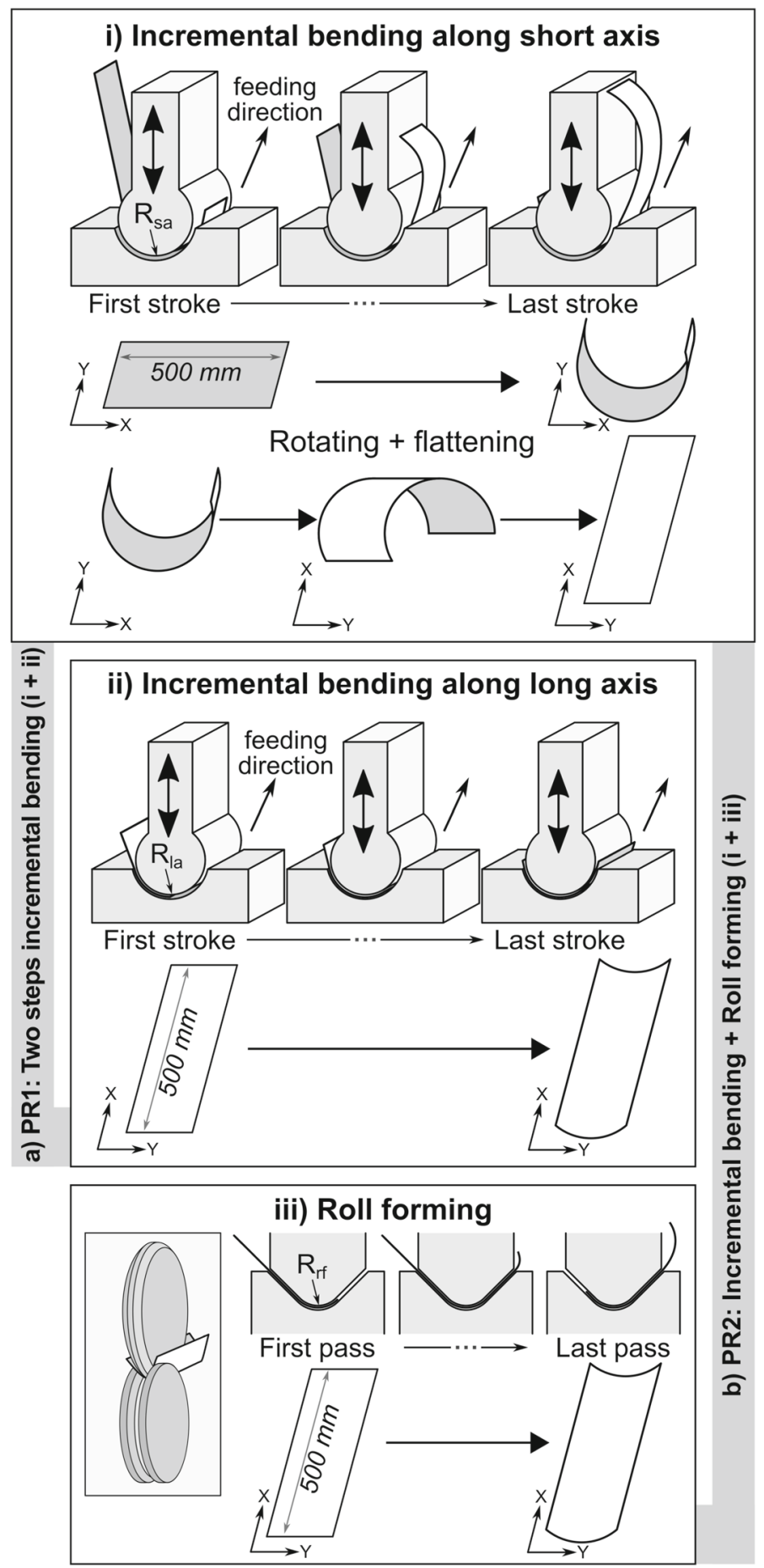

Fig. 2 Used production steps: (i) incremental bending along the short axis with radius $\mathrm{R}_{s a}$, (ii) incremental bending along the long axis with radius $\mathrm{R}_{l a}$, (iii) roll forming with $\mathrm{R}_{r f}$; considered production routes: a PR1 "Two steps incremental bending" (uses step i and ii), b PR2 "Incremental bending and roll forming" (uses step i and iii) 


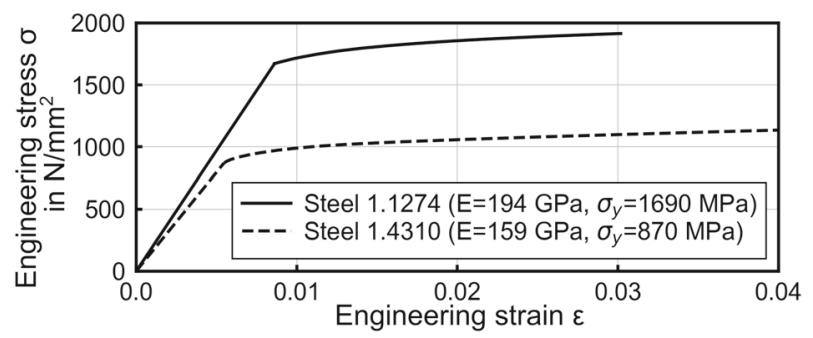

Fig. 3 Stress-strain curves of steels 1.1274 and 1.4310

Table 1 Kinematic parameters of the materials

\begin{tabular}{llr}
\hline Steel & C & $\gamma$ \\
\hline 1.1274 & 37360.04 & 161.73 \\
1.4310 & 24932.94 & 142.05 \\
\hline
\end{tabular}

A simulation study was carried out to analyse the different production routes and various process parameters with regard to the possibility of producing bistable profiles and the corresponding profile radii. Therefore, the FE software Abaqus/Explicit was used for both production routes.

Existing residual stresses from the manufacturing route of the semi-finished product (mainly rolling processes) were not taken into account. The sheet is stress-free at the start of the simulations. The simulation models are constructed as follows: the shell element type S4R with 21 integration points (layers) and a size of $2 \times 2 \mathrm{~mm}^{2}$ are used to mesh the workpieces with the dimension $300 \times 500 \mathrm{~mm}^{2}$ and a thickness of $0.2 \mathrm{~mm}$. The incremental bending simulation mainly consists of the deformable sheet and two rigid dies with certain bending radius. According to bending step size the feeding distance is calculated and the sheet after each bending is pushed forward on the required position.

The roll forming simulation mainly consists of the deformable sheet and a pair of two rigid rollers with certain rolling radius. The stress and strain values of each sheet element after incremental bending simulation are imported to the sheet part in roll forming simulation. For this reason the mesh of the sheet part at incremental bending and roll forming simulation should be equal. After each roll forming step the sheet is flattened using additional rigid parts and the rollers are positioned to the initial position, taking into account the rolling step size and the number of roll forming steps. The subroutine VDISP is used for roller positioning during the roll forming simulation. It should be mentioned, that during simulation of two step processes, the numerical disturbances are accumulated along each process step. Therefore, the simulation results should not be considered as a perfect depiction of the real production processes, but they can be used for an estimation of bistability and profile geometries under different process conditions and for different materials.

All simulations were executed for both materials. Moreover, for describing plastic behaviour of the materials, the "combined isotropic/kinematic hardening model" was used. The kinematic hardening parameters were calculated using Abaqus/CAE by specifying half-cycle test data (i.e. stress-strain curves, see Fig. 3) and are presented in Table 1. The chosen element size and number of integration points is a compromise between calculation time and sufficient precision of residual stress distribution through the sheet thickness. The contact conditions between sheet and rigid tools are described by a "surface-to-surface" approach with a friction coefficient of 0.3 .

To determine the bistability as well as the radii of stable geometries of the deformed sheet an additional FE simulation was developed. After the last step of previous simulations the values of stresses (S), plastic strains (PE) and plastic equivalent strains (PEEQ) of each element of the flattened sheet are read. In the new model the sheet with equivalent size and mesh is created and the values are imported for each corresponding element. After that the sheet is slightly bended in both directions subsequently in order to determine bistability and stable radii.

\subsection{Two steps incremental bending simulation (PR1)}

The whole simulation process is divided into the following steps (Fig. 2a): incremental bending of the sheet along Y-axis in positive Z-direction $\rightarrow$ flattening of the sheet $\rightarrow$ incremental bending of the sheet along X- 


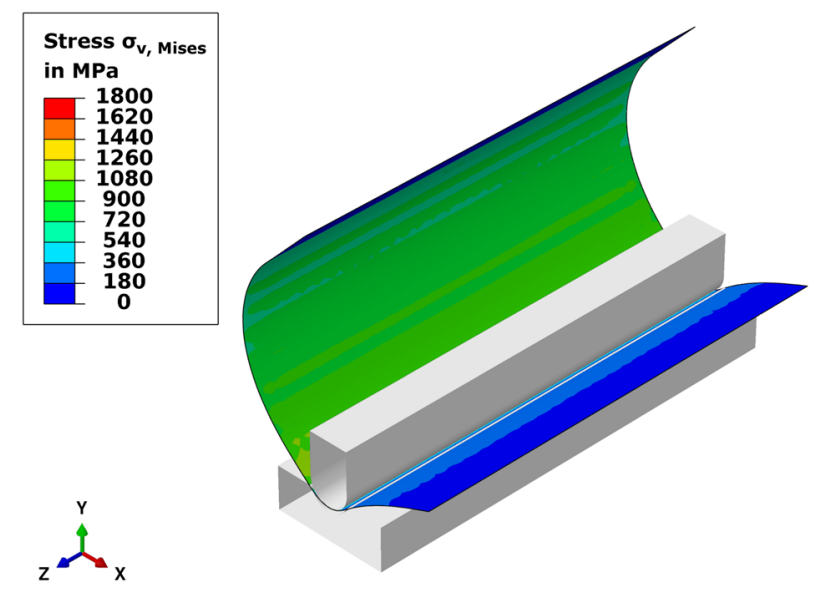

Fig. 4 Depiction of the incremental bending simulation for steel 1.1274

(a)

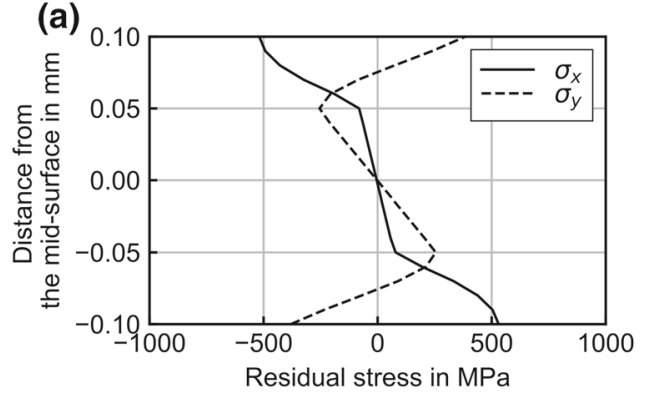

(b)

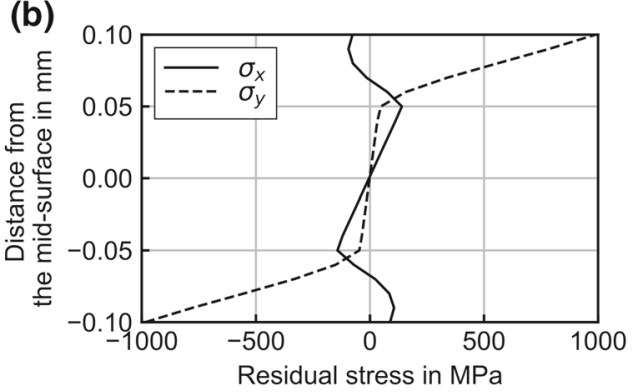

Fig. 5 Residual stresses along the 1.1274 steel sheet cross section in the first (a) and second stable state (b) after simulation of two steps incremental bending (PR1) around $\mathrm{R}_{s a}=\mathrm{R}_{l a}=6 \mathrm{~mm}$

axis in negative Z-direction $\rightarrow$ flattening of the sheet $\rightarrow$ test of bistability by a slight bending of the sheet in positive Z-direction. The feed distance between bending strokes (lateral shift of the sheet) is kept constant and equals the current bending radius. The step size and sheet dimension thus determine the number of bending operations. In the parameter study, the influence of the bending radii $\mathrm{R}_{s a}$ (bending radius along short axis) and $\mathrm{R}_{l a}$ (bending radius along long axis) is examined in a range from 6 to $10 \mathrm{~mm}$. In Fig. 4 the depiction of the incremental bending simulation is shown. Figure 5 shows a representative distribution of residual stresses after two steps incremental bending for material 1.1274 with bending radii $\mathrm{R}_{l a}=\mathrm{R}_{s a}=6 \mathrm{~mm}$ in both stable states.

In Fig. 6 the simulation results (achieved bistability, radii of both stable geometries) are depicted for the steels 1.1274 and 1.4310, respectively. The achieved profile geometries are displayed in a range from 0 to $300 \mathrm{~mm}$. The upper limit is motivated by the used sheet width. In general, for both steel grades bistable properties with different radii of the stable geometries can be achieved. Moreover, various observations can be made: (1) larger first bending radii can be used for the steel 1.1274 compared to 1.4310 in order to achieve bistable properties; (2) steel 1.4310 allows for larger radii within the second bending operation than 1.1274. The reason for these two observations lies in the influence and interaction of parameters such as Young's modulus, yield strength (see Fig. 3), bending radii etc. on the residual stress distribution in the sheet; (3) since the residual stresses introduced in the individual bending operations are superimposed, there are differences in the profile radii achieved depending on the selected bending radii sequence. The results shown can therefore be used to first determine the target radii of the two stable geometries and then to select the corresponding bending radii and the corresponding sequence.

\subsection{Incremental bending and roll forming simulation (PR2)}

In the simulation process of production route PR2 (Fig. 2b) compared to the process route PR1, the second bending step is replaced by roll forming along the same $\mathrm{X}$-axis. The feed distance is kept constant and equals 

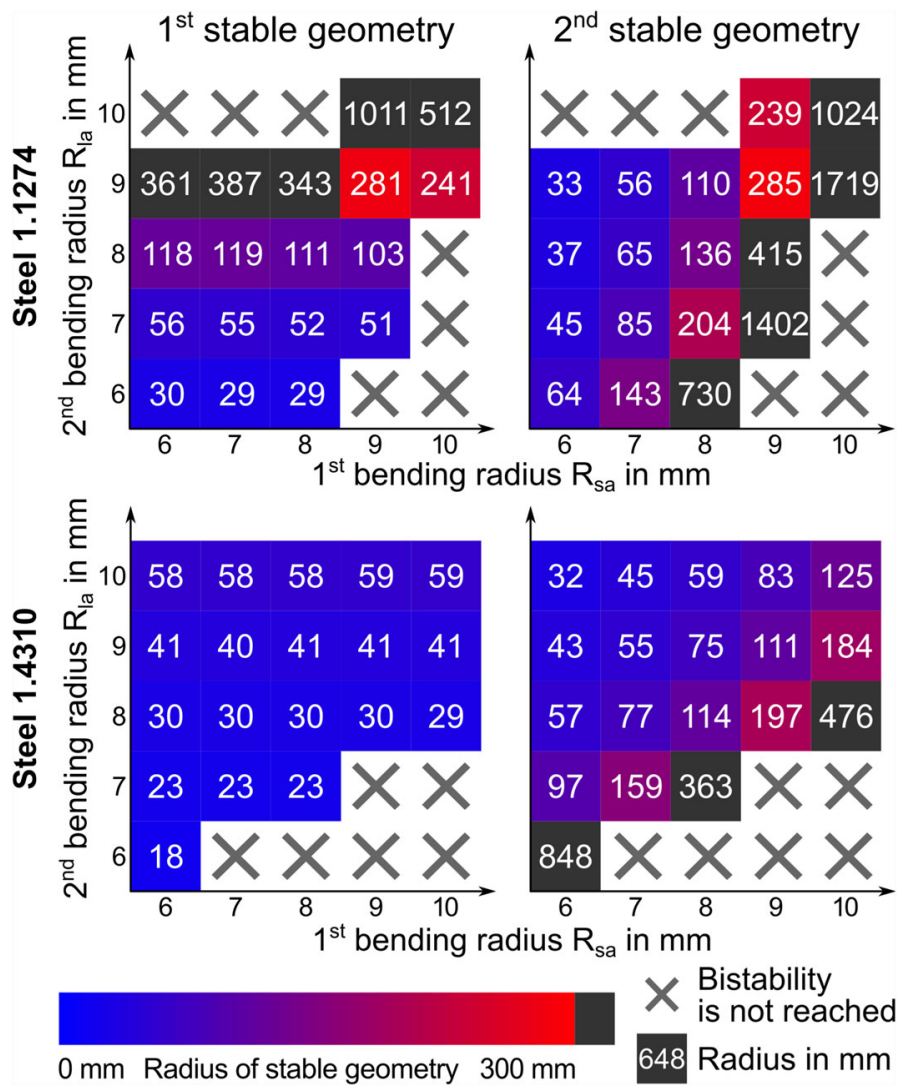

Fig. 6 Heat maps for the achievable bistability and profile geometry in production route (PR1) for steel 1.1274 and 1.4310 obtained after simulations

the current bending or rolling radius, respectively. After each roll forming pass the sheet is flattened and rolling tools are positioned for the next pass. In Fig. 7 the depiction of the roll forming simulation is shown.

In the parameter study the influence of bending radius $\mathbf{R}_{s a}$ in incremental bending and rolling radius $\mathbf{R}_{r f}$ in roll forming is examined in a range from 6 to $10 \mathrm{~mm}$. Figure 8 shows a representative distribution of residual stresses after bending (bending radius $6 \mathrm{~mm}$ ) and roll forming (bending radius of $6 \mathrm{~mm}$ ) for material $1.1274 \mathrm{in}$ both stable states. In Fig. 9 the simulation results (achieved bistability, radii of stable geometries) are depicted for both steels. Bistable properties with different radii of the stable geometries can be achieved for both steels. Furthermore, the results show that: (1) larger radii can be used in incremental bending ( $1^{\text {st }}$ bending) for steel 1.1274 compared to 1.4310 in order to achieve bistable properties; (2) steel 1.4310 allows for larger radii within the roll forming step than 1.1274.

\subsection{Comparison of the production routes}

By comparing the two production routes PR1 (bending - bending) and PR2 (bending-roll forming), recommendations for an experimental production of bistable metallic profiles can be given for both materials under consideration. In general, it must be taken into account that in incremental bending the plastic deformation is incorporated over the entire length of the sheet, whereas in roll forming the plastic zone evolves gradually in the direction of rolling.

For steel 1.4310 bistable profiles can be produced with the same tool radius combinations using both production strategies. In addition, the profile radii achieved are almost identical. The profiles produced by PR1 have a consistently smaller first stable and second stable geometry than profiles produced by PR2.

With the stronger material 1.1274 , the attainability of bistability and profile geometry depends more significantly on the selected production route (PR1 or PR2) and the chosen combination of radii. Such difference could be explained by numerical disturbances during roll forming simulations. During each roll forming pass, a 

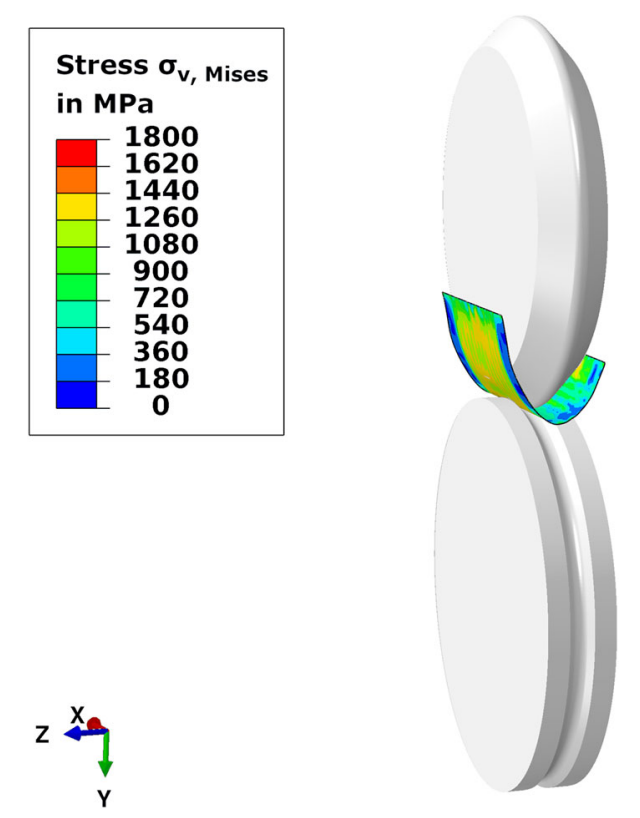

Fig. 7 Depiction of the roll forming simulation for steel 1.1274
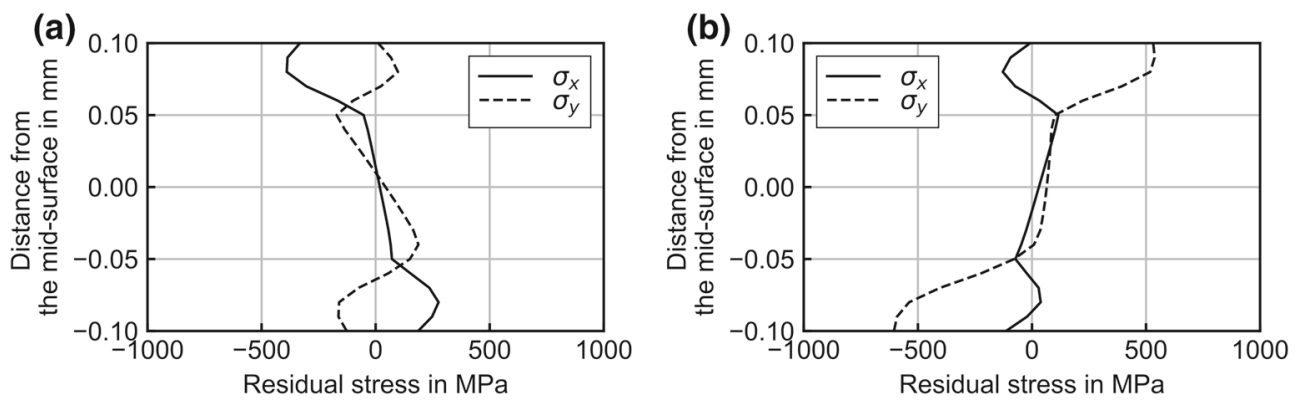

Fig. 8 Residual stresses along the 1.1274 steel sheet cross section in the first (a) and second stable state (b) after simulation of incremental bending and roll forming (PR2) around $\mathrm{R}_{s a}=\mathrm{R}_{r f}=6 \mathrm{~mm}$

certain oscillation of the rolling force occurs, which leads to inhomogeneous distribution of the residual stresses along the sheet length. The PR1 production route enables a bistable profile to be realized with 18 different radius combinations. In contrast, only 11 radius combinations lead to bistable geometries in production route PR2. In addition, the achieved bistable profiles have larger radii of the stable geometry when produced using production route PR2. The reason for this is the significantly different residual stress distribution depending on the selected process route. The comparison of Figs. 5 and 8 clearly shows that greater residual stresses prevail in the edge areas of the sheet produced using PR1. These higher residual stresses increase the occurring bending moment and thus lead to narrower structural radii.

\section{Experimental validation for selected process conditions}

The previously presented simulation study shows that large area bistable profiles with different geometry radii can be produced using both production routes and for both spring steels. Selected radius combinations were tested experimentally to confirm the simulations. The results are presented in the following for both production routes. 

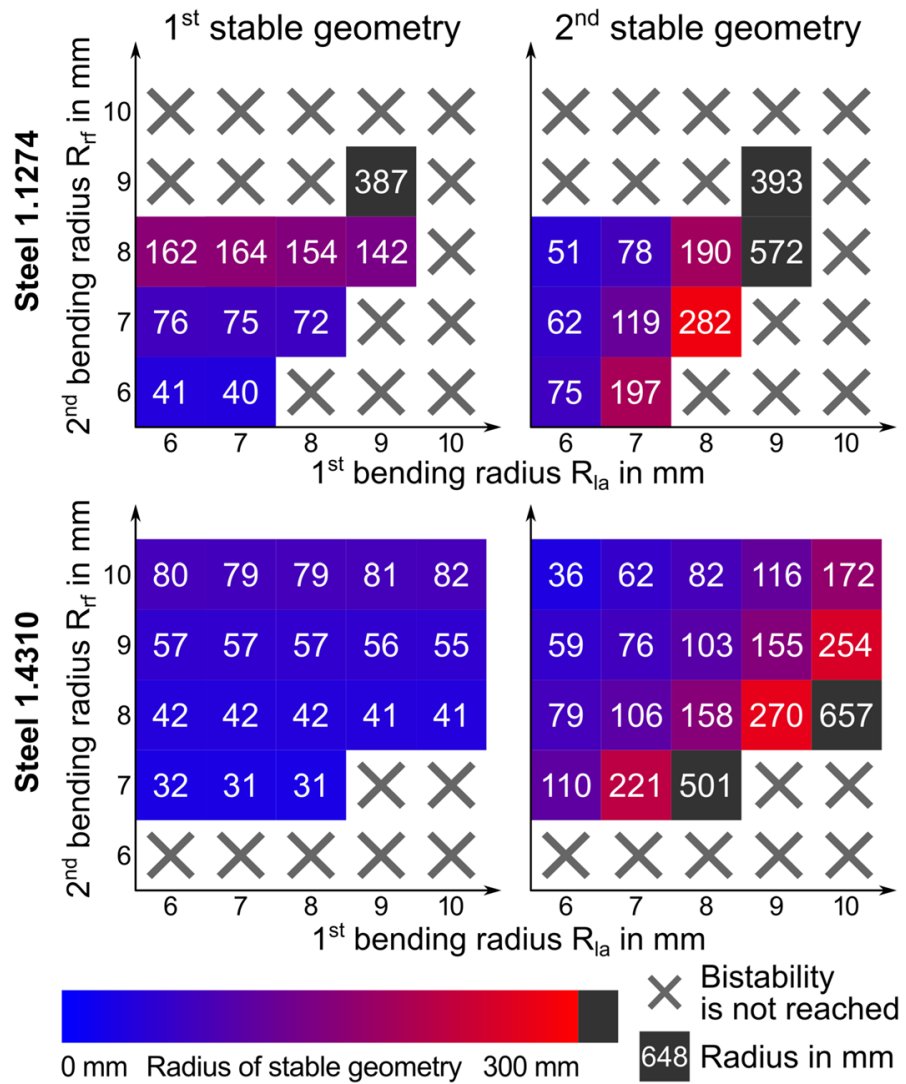

Fig. 9 Heat maps for the achievable bistability and profile geometry in production route PR2 for steel 1.1274 and 1.4310 obtained after simulations

\subsection{Two steps incremental bending experiments (PR1)}

The experiments for production route PR1 were carried out on a Trumpf TrumaBend V50 bending press. Different bending radii can be tested and the effort for tool production is manageable. The sheets used in the experiments were taken from a delivered strip in the as-rolled condition and used without further annealing treatment. The second bending of the sheet is performed in the rolling direction of the strip. In Fig. 10 the experimental results are depicted for the steels 1.1274 and 1.4310 , respectively. The size of the sheet is $300 \times 500 \mathrm{~mm}^{2}$ with a thickness of $0.2 \mathrm{~mm}$. Please note that the bending radius of $9 \mathrm{~mm}$ has not been tested experimentally. Apart from that, an attempt was made to ensure the same production conditions as in the simulation study. The main deviations between the simulations and the experimental investigations are related to the stable geometry radii after springback, where the difference is between $0 \%$ and $172 \%$ (average $62 \%$ ) for steel 1.1274 and between 5\% and $134 \%$ (average $47 \%$ ) for steel 1.4310 . These differences are probably based, on the one hand, on the simplifications within the simulations (e.g. using of "combined isotropic/kinematic hardening model", no initial residual stresses in the sheet, mesh size) and, on the other hand, on the predominantly kinematic deviations between experiment and simulation.

In addition, some combinations of bending radii during experiments do not lead to bistability as during simulations. During experiments, in order to achieve a bistable profile, the second bending radius should not be smaller than the first one. It is also remarkable that, in contrast to the simulations for 1.1274, bistability for radii combinations with $\mathrm{R}_{l a}>R_{s a}$ is achieved in experiments (e.g. $6 \mathrm{~mm}, 10 \mathrm{~mm}$ combination). Nevertheless, the following dependence applies to simulations and experiments: if the first bending radius is constant, an increasing second bending radius results in an increase of the radius of first and a decrease of the radius of second stable geometry.

Since the experiments were carried out with a manual feed, no exact and constant feed distance as in the simulations can be guaranteed. Nevertheless, the basic tendencies between simulation and experiment coincide and, as shown in Fig. 11a, b, large area bistable and fully closed metallic profiles, which have a coiled transport 

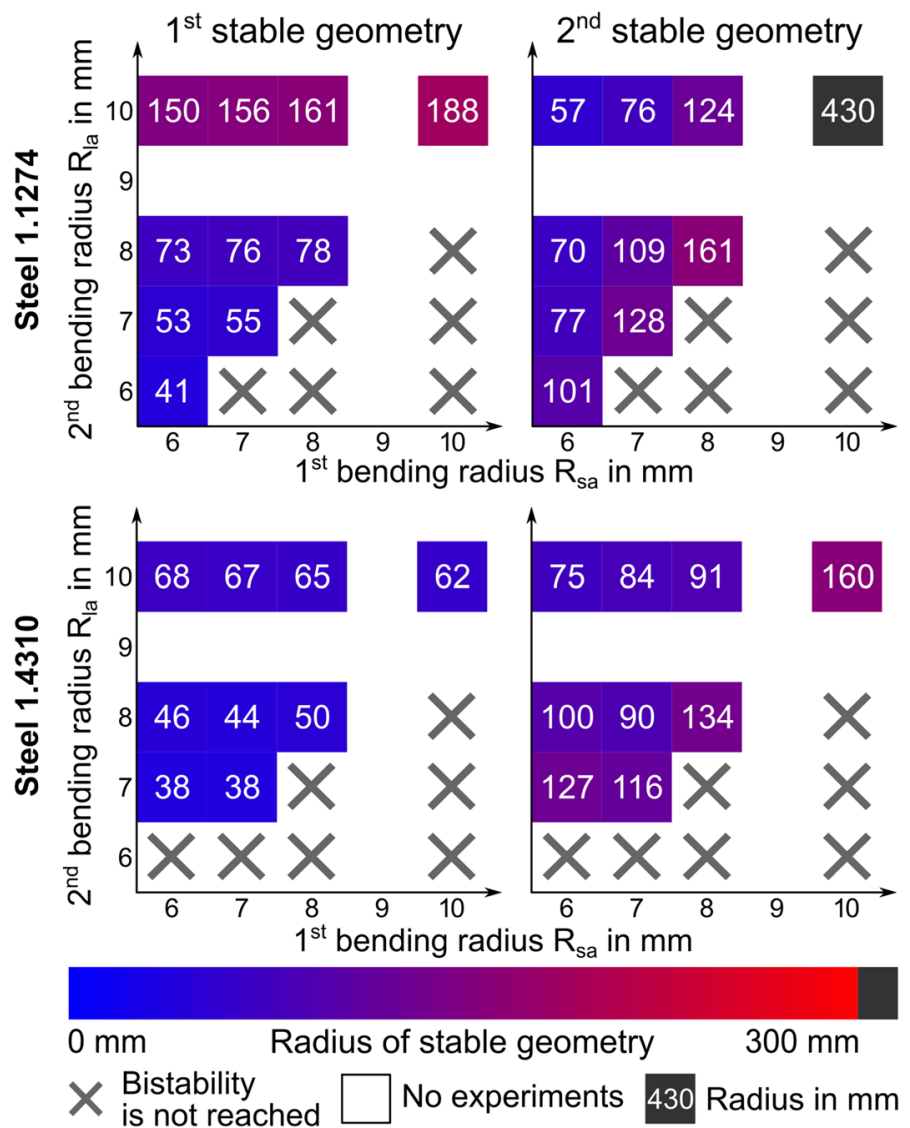

Fig. 10 Heat maps for the experimentally achieved bistability and profile geometry in production route PR1 for steel 1.1274 and 1.4310

and an unfolded geometry with even $1000 \mathrm{~mm}$ length, can be produced using production route PR1. For this purpose, sheets with dimensions of $1000 \times 300 \mathrm{~mm}^{2}$ were used.

\subsection{Incremental bending and roll forming experiments (PR2)}

Since the manufacturing effort for rolls with different radii is much greater, only the combination with one bending radius of $6 \mathrm{~mm}$ in roll forming (2nd process step in PR2) was tested experimentally. For this purpose, a Dreistern rolling mill was equipped with a corresponding pair of rolls and the sheet was rolled several times. In this process step the rolling direction of roll forming and the rolling direction from the strip production are equal. The positioning of the sheet is a major challenge in roll forming. Particularly after rolling passes have already been made, parallel guidance of the sheet under elastic tension is difficult. Nevertheless, bistable profiles were successfully produced via process route PR2. Since no major experimental parameter study can be carried out for the reasons mentioned earlier, only a representatively selected bistable profile with different geometric radii is shown in Fig. 11c. Hence, large area bistable metallic profiles, which have a coiled transport geometry and an unfolded structure, could also be produced with production route PR2.

\section{Summary}

Although the results of simulation and experiments differ from each other, nevertheless, the developed models greatly facilitate the task of finding working combinations of bending radii for production of bistable sheets. After finding such combinations by roll forming simulations the number of rolls to be produced with the required bending radius has been significantly reduced. The simulation results also show that the radii of stable 


\section{(a) \\ (b)}

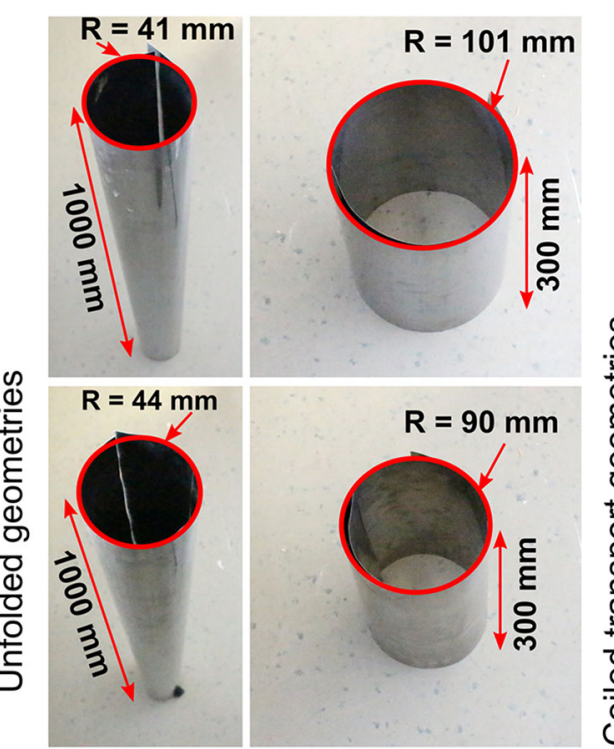

(c)

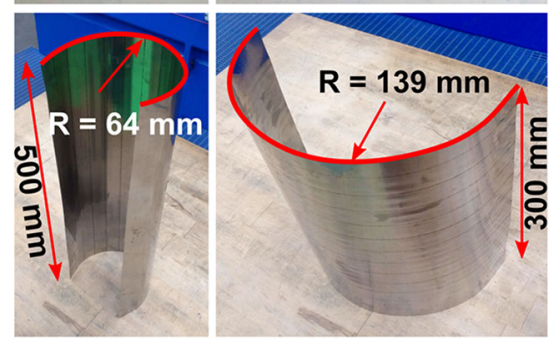

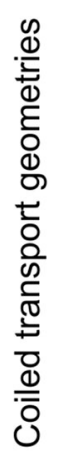

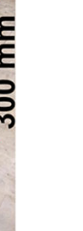

Fig. 11 Selected produced bistable profiles in a PR1 with 1.1274 and bending radii combination ( $6 \mathrm{~mm}, 6 \mathrm{~mm})$, b PR1 with 1.4310 and bending radii combination $(7 \mathrm{~mm}, 8 \mathrm{~mm})$, c PR2 with 1.1274 and bending radii combination $(6 \mathrm{~mm}, 6 \mathrm{~mm})$

states after bending and rolling of a sheet, even with the same bending radii, is significantly different. This can help design a working continuous bistable sheet production process in the future.

The present work has clearly shown that large area bistable metallic profiles with various stable geometries can be produced in different production routes and with different materials: (1) PR1 "Two steps incremental bending", (2) PR2 "Incremental bending and roll forming". However, the results also show that the process routes have different limitations depending on the desired profile geometry. With PR1, fully closed bistable profiles can be effectively produced on a single machine. However, the length of the unfolded structure is limited to the available tool length. On the other hand, PR2 can be used to create bistable profiles of any length. The handling is, however, difficult within the roll forming process and it has not yet been possible to create fully closed profiles. The reason for this is the changed residual stress profile between PR2 and PR1.

Acknowledgements The authors thank the Deutsche Forschungsgemeinschaft (DFG, German Research Foundation) for funding within the priority program SPP 2013 "The utilization of residual stresses induced by metal forming" (project HI 790/57-2, No. 374688658). Moreover, the authors like to thank Mrs. Yun Xie for her support in developing FE-models.

Funding Open Access funding enabled and organized by Projekt DEAL.

Open Access This article is licensed under a Creative Commons Attribution 4.0 International License, which permits use, sharing, adaptation, distribution and reproduction in any medium or format, as long as you give appropriate credit to the original author(s) and the source, provide a link to the Creative Commons licence, and indicate if changes were made. The images or other third party material in this article are included in the article's Creative Commons licence, unless indicated otherwise in a credit line to the material. If material is not included in the article's Creative Commons licence and your intended use is not permitted by statutory regulation or exceeds the permitted use, you will need to obtain permission directly from the copyright holder. To view a copy of this licence, visit http://creativecommons.org/licenses/by/4.0/. 


\section{References}

1. Totten, G., Inoue, T., Howes, M.: Handbook of residual stress and deformation of steel, Chap, 10. In: Residual Stress in the Forming of Materials, pp. 141-149 (2002)

2. Tekkaya, A., Gerhardt, J., Burgdorf, M.: Residual stresses in cold-formed workpieces. CIRP Ann. 34(1), 225-230 (1985)

3. Kebadze, E., Guest, S., Pellegrino, S.: Bistable prestressed shell structures. Int. J. Solids Struct. 41, 2801-2820 (2004)

4. Wolf, G., Wolf, M.: German patent application DE202006004427U1: Metallband. Cobra Bandstahl GmbH. (2006)

5. https://www.duo-shop.de/de-DE/Details/Brunnen-Roll-Lineal-30-cm-Snap-it/18018

6. Pavliuchenko, P., Teller, M., Grüber, M., Bremen, T., Hirt, G.: Production of bistable fully closed metallic shells by introducing residual stresses during bending processes. Prod. Eng. 13(2), 201-209 (2019)

7. Ona, H.: Cold roll forming for high tensile strength steel sheet proposition on forming of thin spring steel sheet pipe. J. Mater. Process. Technol. 153-154, 247-252 (2004)

8. Groskopfs, E.: US patent application US3434674A: Storable tubular extensible member device. De Havilland Aircraft of Canada Ltd. (1967)

9. Pellegrino, S.: Deployable structures, Chapter 1: Deployable structures in engineering. Springer, Vienna (2001)

10. Pavliuchenko, P., Teller, M., Grüber, M., Hirt, G.: A semianalytical model for the determination of bistability and curvature of metallic cylindrical shells. J. Manuf. Mater. Process. 3(22), 1-16 (2019)

Publisher's Note Springer Nature remains neutral with regard to jurisdictional claims in published maps and institutional affiliations. 